\title{
Biological activity of a peptidoglycan extracted from Leptospira interrogans: in vitro studies
}

\author{
Marina Cinco, ${ }^{1}$ Sandra Perticarari,${ }^{2}$ Gianni Presani,${ }^{2}$ Aldo Dobrina ${ }^{3}$ and Franco Liut ${ }^{4}$ \\ ${ }^{1}$ Istituto di Microbiologia, Università di Trieste, via Fleming 22, 34100 Trieste, Italy \\ ${ }^{2}$ IRCCS Burlo Garofolo, Trieste, Italy \\ ${ }^{3}$ Istituto di Patologia Generale, Università di Trieste, 34100 Trieste, Italy \\ ${ }^{4}$ Dipartimento di Biochimica Biofisica e Chimica delle Macromolecole
}

(Received 9 June 1993; revised 29 July 1993; accepted 19 August 1993)

\begin{abstract}
Peptidoglycan (PG) has been isolated from some species of spirochaetes, including Leptospira interrogans. Although leptospiral PG has been chemically characterized, no study has been carried out on its potential biological activity. Since PG of Treponema and Borrelia is biologically active both in vivo and in vitro, we investigated the capacity of a leptospiral PG preparation to induce relevant biological effects. PG extracted from L. interrogans strain Teramo was mitogenic at $0.1 \mathrm{~g} \mathrm{~m} \mathrm{~m}^{-1}$ for human peripheral blood mononuclear cells (PBMC) since it increased the PBMC fraction positive for Ki-67, an antigen expressed by human proliferating cells; at $4 \mu \mathrm{g} \mathrm{ml}^{-1}$, PG was able to induce complement consumption and to stimulate leucocyte phagocytosis and the metabolic burst of resting as well as phagocytosing leucocytes. These findings indicate that Leptospira PG may play a role in modulating the immunocompetent cell functions and suggest that PG can contribute to the host response during Leptospira infection.
\end{abstract}

\section{Introduction}

The presence of cell wall peptidoglycans in the Spirochaetales has been demonstrated for some genera and species, e.g. Borrelia duttoni, Borrelia burgdorferi (Ginger, 1963; Beck et al., 1990), Spirochaeta stenostrepta, Spirochaeta litoralis (Joseph et al., 1973), Reiter Treponema, Treponema pallidum (Pillot \& Reiter, 1965; Umemoto, 1981) and Leptospira (Ginger, 1963; Azuma, 1975). Chemical analysis revealed that the structure of spirochaete peptidoglycans was of the $\mathrm{A}_{2}$-type, according to the classification of Schleifer \& Kandler (1972), and that the diamino acid involved in cross-linking was ornithine in Spirochaeta, Borrelia and Treponema, or diaminopimelic acid for strains representative of the genera Leptospira ( $L$. interrogans and L. biflexa) and Leptonema (Yanagihara et al., 1984). Peptidoglycans and muramyl peptide derivatives have diverse and complex biological effects in mammals, including stimulation of cells involved in the immune response, activation of the

*Author for correspondence. Tel. +3940571081 ; fax +3940 567862 .

Abbreviations: HEC, human epithelial cells; LPS, lipopolysaccharide; PBMC, peripheral blood mononuclear cells; PG, peptidoglycan; PMN, polymorphonuclear leucocytes. complement cascade, adjuvant effect, induction of inflammation, activation of monocytes and neuropharmacological activity (Kotani et al., 1986; StewartTull, 1980). Some of these biological activities have been observed in ornithine A-type PG preparations of $B$. burgdorferi (Beck et al., 1990) and T. pallidum (Umemoto et al., 1981). However, no study has been carried out on the biological effects induced by Leptospira $\mathrm{PG}$ which, in contrast to the other spirochaetes, is pimelic $\mathrm{A}_{2}$-type (Yanagihara et al., 1984).

Recently, we found that whole cell preparations of Leptospira strains were able to elicit a significant proadhesive effect in human endothelial cells (HEC) towards neutrophils, which may represent the first step in vasculitis (A. Dobrina and others, unpublished data). The leptospiral component responsible for this effect was demonstrated to reside in a PG preparation, whereas no activity was found in a Leptospira LPS preparation. Since LPS of Gram-negative bacteria is known to induce the proadhesive activity on HEC besides a variety of other biological effects (Schleimer \& Rutledge, 1986), we questioned whether peptidoglycans were responsible for further biological activities induced in the host by pathogenic leptospires. Therefore we investigated the capacity of leptospiral PG to trigger relevant biological effects, such as complement activation, stimulation of the 
neutrophil and monocyte phagocytosis and metabolic burst, and lymphocyte mitogenicity.

\section{Methods}

Leptospira strain and cultivation. The Leptospira interrogans strain used in this study was Teramo, which we classified (unpublished data) as serovar copenhageni, serogroup Icterohaemorrhagiae, from the Leptospira Reference Collection of our Institute. Leptospires were grown in liquid EMJH medium (Johnson et al., 1973), up to a density of about $10^{8}$ cells $\mathrm{ml}^{-1}$.

Peptidoglycan extraction. PG was extracted according to the procedure of Umemoto et al. (1981). Briefly, 5 litres of leptospire culture were harvested by centrifugation at $10000 \mathrm{~g}$ for $30 \mathrm{~min}$, washed

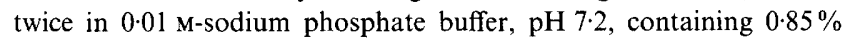
$(\mathrm{w} / \mathrm{v}) \mathrm{NaCl}$ (PBS), and resuspended in $100 \mathrm{ml} 1 \%$ (w/v) SDS in distilled water. The suspension was gently shaken at $37^{\circ} \mathrm{C}$ for $18 \mathrm{~h}$ and then centrifuged at $110000 \mathrm{~g}$ for $60 \mathrm{~min}$. After a second treatment with $1 \%(\mathrm{w} / \mathrm{v})$ SDS the pellet was washed four times with $6 \mathrm{M}$-urea, resuspended in $200 \mathrm{ml}$ distilled water and centrifuged at $1500 \mathrm{~g}$ for $10 \mathrm{~min}$. The supernatant was collected and centrifuged at $110000 \mathrm{~g}$ and the resulting pellet was suspended in $14.6 \mathrm{ml} 0.01 \mathrm{M}-\mathrm{Tris} / \mathrm{HCl}$ buffer, $\mathrm{pH} 7 \cdot 4$, containing $1.46 \mathrm{mg}$ trypsin; the reaction mixture was incubated at $37^{\circ} \mathrm{C}$ for $18 \mathrm{~h}$, washed with distilled water by centrifugation at $110000 \mathrm{~g}$ for $90 \mathrm{~min}$ and then digested with $1.46 \mathrm{mg}$ pronase (Sigma) in

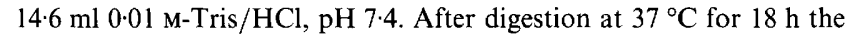
pellet was recovered by centrifugation and then lyophilized. The amount of PG extracted was $0.02 \%(w / w)$ of the dry weight of intact spirochaetes.

Amino acid analysis of the sample was carried out by gas chromatography, following the method of MacKenzie \& Tenashuk (1979). Ornithine was used as an internal standard. Assays were performed on a HP 5890 gas chromatograph supplemented with an HP Ultra 1 capillary column. The analysis revealed a relative molar ratio of the most abundant amino acids, alanine, glutamic acid, glycine and meso-aminopimelic acid, of 0.8, 0.6, 0.66 and 0.62, respectively. Serine, arginine, asparagine, leucine, valine were also detected, but accounted for a very small percentage of the amino acids. This molecular composition was indicative of the presence of a $L$. interrogans $P G$, as already described (Yanagihara et al., 1984). A $10 \mu \mathrm{g}$ sample of PG was checked for the presence of LPS by SDS-PAGE and silver staining according to the method of Tsai \& Frasch (1982), which detects less than $5 \mathrm{ng}$ of rough LPS; no contamination by LPS was found. Moreover, the sample did not give any clotting activity in the Limulus test (Limusate; Haemachem Inc.), which is sensitive to endotoxin in the order of picograms $\mathrm{ml}^{-1}$ up to a dilution of 10 nanograms $\mathrm{ml}^{-1}$.

Haemolytic assay for complement activation by $P G$. The method described by Harrison \& Lachmann (1986) was followed using a pool of fresh human sera as source of complement. Veronal-buffered saline (pH 7.2), as described by Harrison \& Lachmann (1986), was employed for the haemolytic assay; EDTA-containing PBS (pH 7.4) was made with $0.15 \mathrm{M}-\mathrm{NaCl}, 5 \mathrm{~mm}$-sodium phosphate, and $10 \mathrm{~mm}$ EDTA and was employed to block the lytic reaction at the end of the incubation time. Mixtures of $200 \mu \mathrm{l}$ of Veronal-buffered saline containing 1/20 normal serum and various amounts of PG were added to $50 \mu \mathrm{l}$ of sensitized sheep erythrocytes $\left(3 \times 10^{8}\right.$ cells ml $\left.^{-1}\right)$ according to Harrison \& Lachmann (1986) and incubated for $30 \mathrm{~min}$ at $37^{\circ} \mathrm{C}$. The extent of erythrocyte lysis was measured at $415 \mathrm{~nm}$ after the addition of $1 \mathrm{ml}$ icecold PBS-EDTA. The results of two repeated assays were expressed as $\mathrm{CH} 50$ units (ml serum $)^{-1}$, where $1 \mathrm{CH} 50$ Unit was defined as that serum activity able to produce $50 \%$ haemolysis according to the Mayer technique described in the same reference.
Peripheral blood mononuclear cells (PBMC) preparation. Mononuclear cells were isolated from heparinized blood of healthy donors by Ficoll-Hypaque separation (Seromed), then washed and resuspended at a final concentration of $5 \times 10^{5} \mathrm{cell} \mathrm{ml}^{-1}$ in RPMI-1640 (Seromed), supplemented with $2 \%$ of the synthetic serum Ultroser G (GibcoBRL), antibiotics and glutamine. Cultures were incubated at $37^{\circ}$ for $48 \mathrm{~h}$ in the presence of $E$. coli LPS (Sigma) or PG at $0.1,1$, or $10 \mu \mathrm{g} \mathrm{ml}^{-1}$. After the incubation, supernatants were removed by centrifugation at $750 \mathrm{~g}$.

Mitogenic assay: antigen Ki-67 measurement. Ki-67 antigen is expressed by all human proliferating cells, preferentially during the $\mathrm{G}_{1}-\mathrm{G}_{2}$ phases and not in $\mathrm{G}_{0}$ (Gerdes et al., 1984). A fluoresceinisothiocyanate (FITC)-conjugated monoclonal antibody to $\mathrm{Ki}-67$ (DAKO-Ki-67; Dakopatts) detects the amount of this antigen expressed by proliferating cells (Gerdes et al., 1984) in a cell suspension. Flow cytometric analysis of fluoresceinated $\mathrm{Ki}-67$ was performed to measure the growth fraction of cell populations with the same sensitivity as assay of $\left[{ }^{3} \mathrm{H}\right]$ thymidine uptake (Schwarting et al., 1986). After exposure to various amounts of LPS and PG, PBMC were fixed with a paraformaldehyde/lysine/periodate-solution for $30 \mathrm{~min}$ at $40^{\circ} \mathrm{C}$. Paraformaldehyde/lysine/periodate solution was prepared according to the method described by McLean \& Nakane (1974). The preparation was then washed, resuspended in PBS and stained with FITC-conjugated DAKO-Ki-67 monoclonal antibody, as described by the manufacturer (Dakopatts). Fluorescence intensity was read by flow cytometry and expressed as the percentage of proliferating cells (Schwarting et al., 1986). Experiments were repeated in triplicate.

Assay for the simultaneous quantification of phagocytosis and oxidative products. We used the method described elsewhere (Perticarari et al., 1991), with minor modifications. Briefly, leucocytes were isolated by dextran sedimentation of heparinized blood samples (5 IU heparin $\mathrm{ml}^{-1}$ ) obtained from healthy volunteers. After centrifugation at $100 \mathrm{~g}$ for $10 \mathrm{~min}$ of the leucocyte-rich plasma, the remaining erythrocytes were lysed with $0.8 \%(\mathrm{w} / \mathrm{v}) \mathrm{NH}_{4} \mathrm{Cl}$. The pellet was washed once and resuspended in Hanks' balanced salt solution (HBSS; Seromed), and resuspended in the same medium. FITC-labelled Staphylococcus aureus cells, prepared as described previously (Perticarari et al., 1991), were opsonized with a pool of fresh human sera and used at a concentration of $10^{8}$ cells $\mathrm{ml}^{-1}$ in HBSS. For the assay, $100 \mu \mathrm{l}$ HBSS containing $5 \times 10^{5}$ leucocytes were incubated for $15 \mathrm{~min}$ with $100 \mathrm{ng}$ hydroethidine $\mathrm{ml}^{-1}$ (HE; Soc. Italiana Chimici), which was converted to the red fluorescent ethidium bromide in the presence of oxidative products. Bioassays were performed in the presence or absence of $100 \mu \mathrm{l}$ fluoresceinated bacteria and in the presence or absence of $15 \mu \mathrm{g}$ LPS or $20 \mu \mathrm{g}$ PG. After incubation, $500 \mu \mathrm{l}$ cold PBS and $100 \mu \mathrm{l}$ $0.25 \mathrm{~g}$ crystal violet $1^{-1}$ were added to each tube to quench the fluorescence of non-ingested bacteria. Two independent experiments were performed.

Flow cytometry. Analyses of fluorescence were done by using a FACSscan flow cytometer (Becton-Dickinson). Ten-thousand events per sample were acquired in the list mode by linear amplification for forward (FSC) and side scatter (SSC) signals. Fluorescence signals were then logarithmically amplified. Data were analysed by a Consort 32 with LYSIS II software. Consort 32 is a laboratory data analysis system used with the FACScan to perform data acquisition and analysis on cell samples. It uses Hewlet-Packard hardware with the Pascal operating system. Leucocyte populations were selected as R1 and R2 regions for polymorphonuclear leucocytes (PMN) and monocytes, respectively, on the basis of FSC and SSC properties. Measures of phagocytosis and of the oxidative burst were performed by two colour analysis: green fluorescence (FL1) indicating the rate of phagocytosis, and red fluorescence (FL2) the rate of production of oxidative metabolites (Perticarari et al., 1991). Compensations for FL1 to FL2 and FL2 to FLl were $6 \%$ and $30 \%$, respectively. 


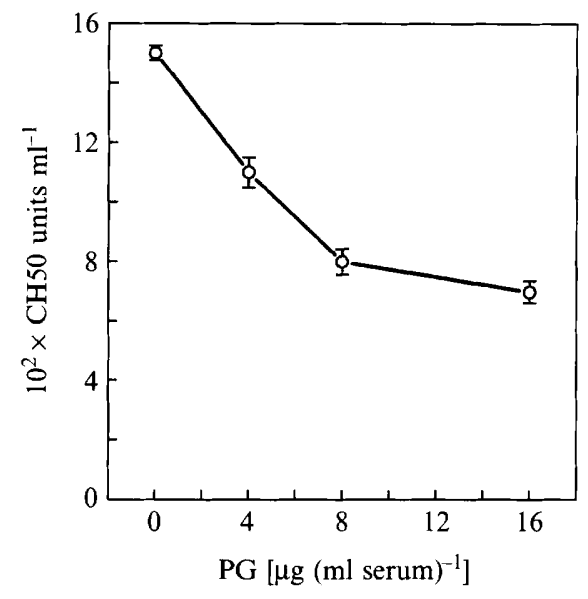

Fig. 1

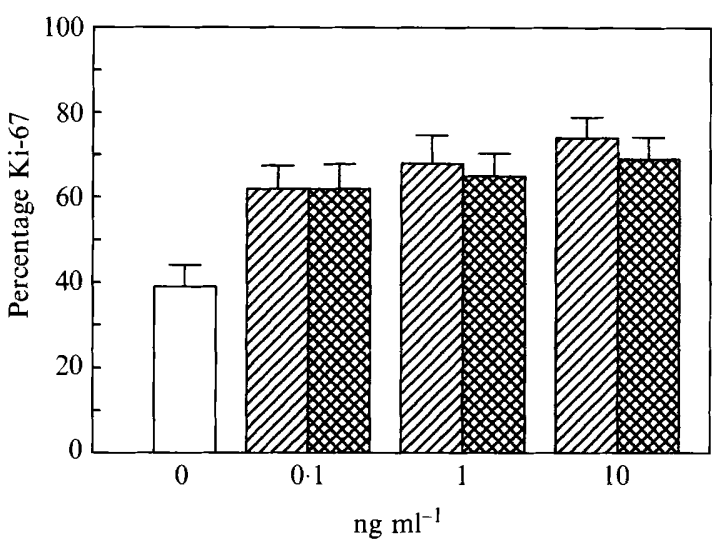

Fig. 2

Fig. 1. Complement activation by PG. Values of CH50 are means \pm SD of three independent assays $\left(15 \mathrm{CH} 50 \times 10^{2} \mathrm{ml}^{-1} \mathrm{corresponded}^{-}\right.$ to $50 \%$ haemolysis by distilled water).

Fig. 2. Cell fraction of PMBC preparations stimulated by PG or E. coli LPS. Values represent the percentage of Ki-67-antigen-positive cells detected by flow cytometry, and are means \pm SD of three independent assays. $\square$, Medium; $\square$, PG; $\otimes$, LPS.

\section{Results}

Activation of complement by leptospira $P G$

Fig. 1 shows the degree of complement consumption triggered by PG in normal human serum. It can be seen that PG, though already effective at $4 \mu \mathrm{g} \mathrm{ml}^{-1}$ induces an almost $50 \%$ depletion of complement activity at $8 \mu \mathrm{g} \mathrm{ml}^{-1}$.

\section{Mitogenic activity on PBMC by Leptospira PG}

The mitogenic activity of PG was determined by measuring the amount of the $\mathrm{Ki}-67$ antigen expressed on PBMC after exposure to PG or LPS. This determination identifies proliferating cells in a PBMC population. After incubation for $2 \mathrm{~d}$, PG was able to induce a dosedependent cellular response that was at least as high as the corresponding effect triggered by $E$. coli LPS (Fig. 2). In fact, at $1 \mu \mathrm{g}$ about $69 \%$ of the cells underwent multiplication, compared to $62 \%$ of the PBMC population stimulated by LPS and to the $35 \%$ growth fraction observed in unstimulated cells.

Stimulation of phagocytosis and of the respiratory burst of PMN and monocytes

Fig. 3 shows one of the two experiments performed on the effect of PG on phagocytosis and on the metabolic activation of PMN and monocytes. The rate of phagocytosis of fluoresceinated Staph. aureus is represented by a shift of green fluorescence along the abscissa, from quadrant 3 to 4 ; the respiratory burst is indicated by an increase of red fluorescence along the ordinate, from quadrant 4 to 2 . The fraction of stimulated cells is expressed in percentage values in Table 1. When PMN were preincubated with $\mathrm{PG}$, two effects were observed: (1) induction of the metabolic activation on resting cells (plot $c$ ) followed by amplification of this effect during phagocytosis, and (2) an increase in the number of phagocytosing cells, as indicated by the shift of contour plots towards square 4 (plot $f$ ). Percentage values of metabolic activation observed after incubation with PG in two experiments were higher than those observed after exposure to E. coli LPS, since PG directly stimulated $36 \%$ of the resting and $83 \%$ of the phagocytosing PMN, whereas $8 \%$ and $73 \%$ of the corresponding PMN populations were stimulated by LPS (Table 1).

A metabolic stimulation by PG was also observed on the monocyte population (plots $h-n$ ). Moreover, during phagocytosis (plot $n)$ it was greater $(70 \%)$ than that induced by LPS ( $57 \%)$.

\section{Discussion}

Clinical features of leptospirosis-like haemorrhages, fever and inflammatory symptoms were postulated in the past to be caused by an endotoxin released from leptospires during the infection (Alston \& Broom, 1958). However, though several attempts have been made to demonstrate leptospiral endotoxicity, only limited biological effects were observed in various Leptospira LPS preparations, not sufficient to justify the complex and severe development of the inflammatory response of the host (Arean et al., 1964; Shimizu et al., 1987; Cinco et 

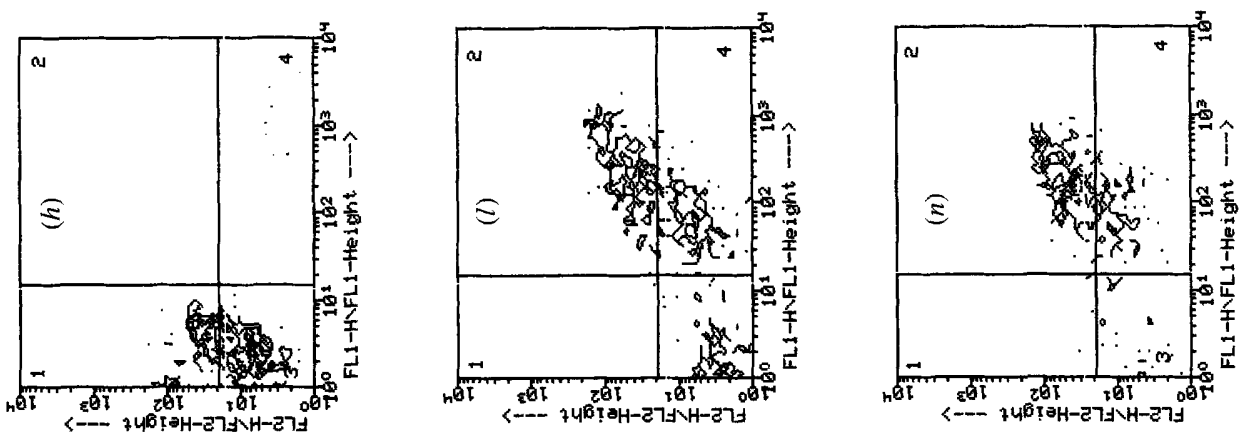

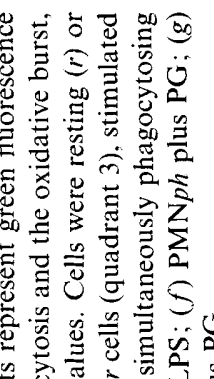

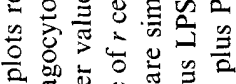

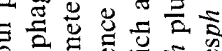

일
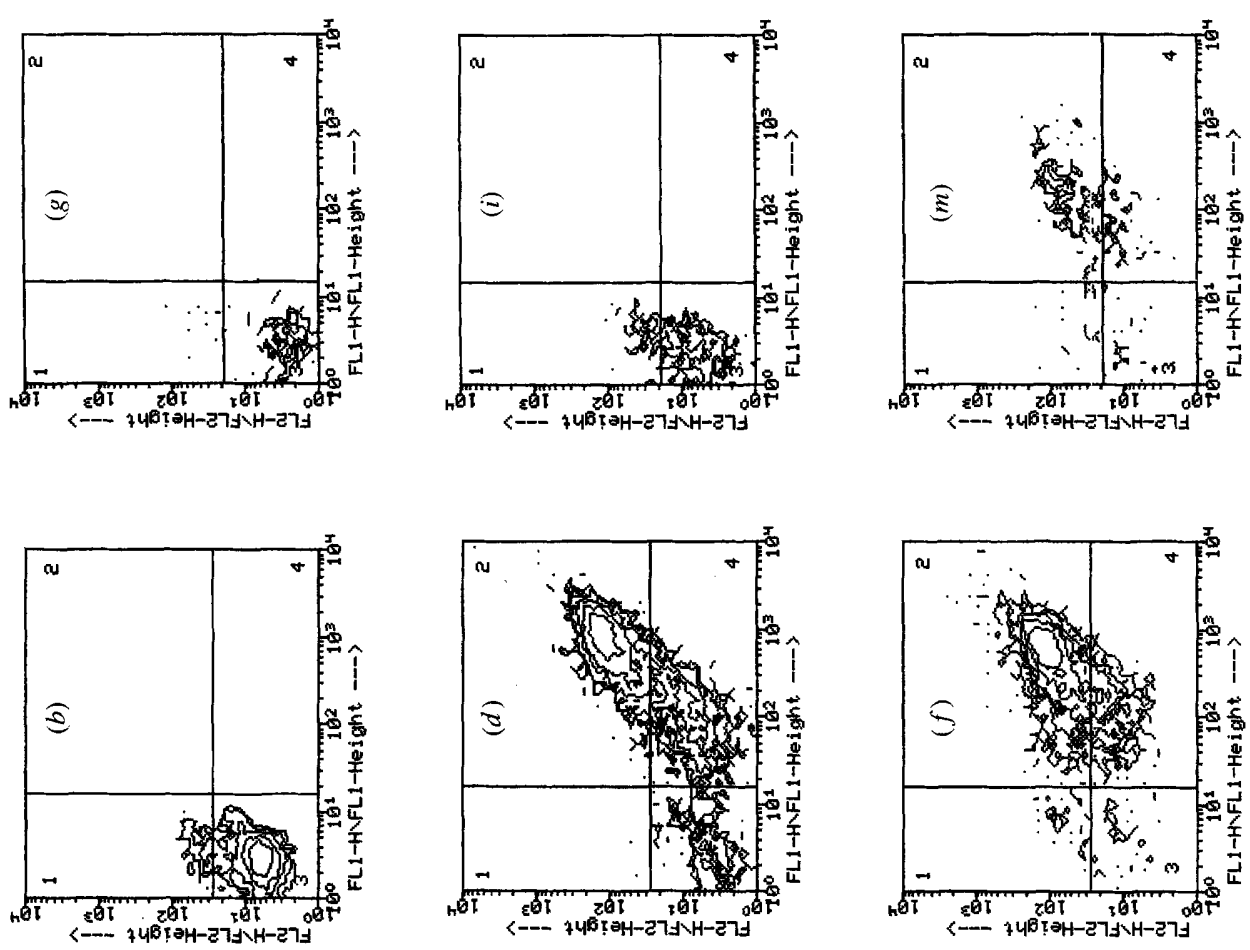

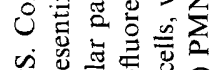

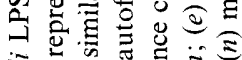

ธิ

4

올

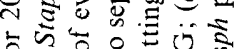

$0: 5$ 등

on

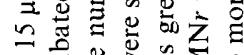

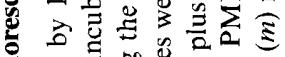

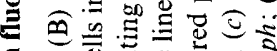

选

㻤

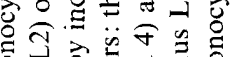

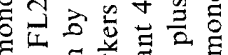

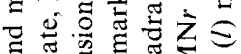

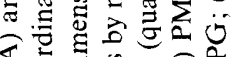

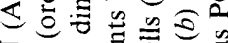

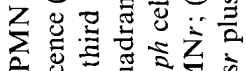

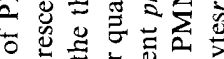

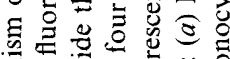

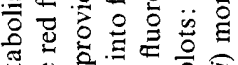

讨

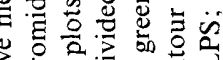

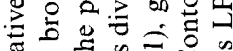
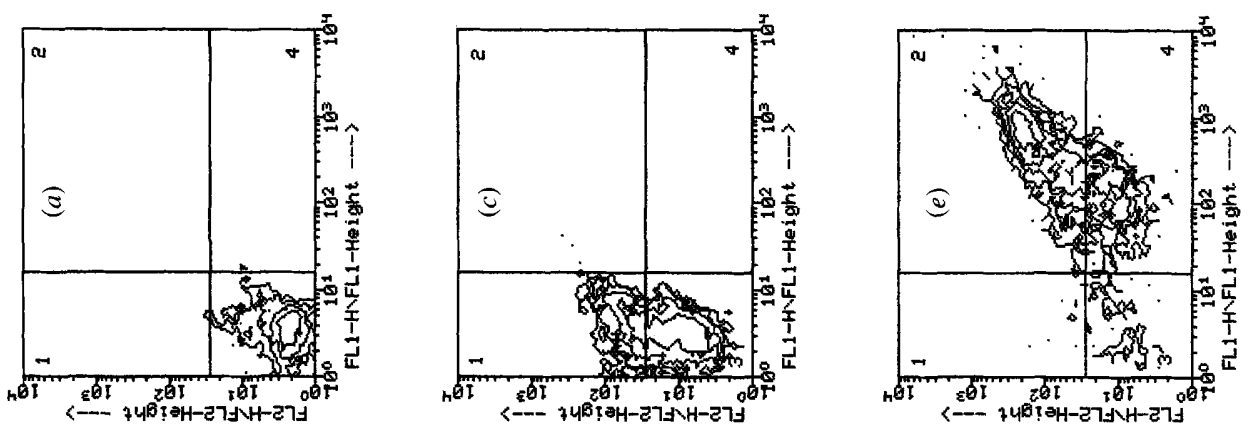

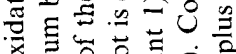

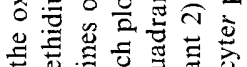

o

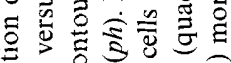

的 300

I

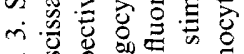

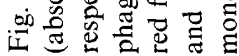


Table 1. Metabolic stimulation of PMN and monocytes after incubation with $P G$ or $E$. coli $L P S$

Values indicate the percentage fraction of the cell population emitting fluorescence: $100 \%$ of PMN was $4.7 \times 10^{6}$ cells and of monocytes $2.9 \times 10^{5}$ cells ml $^{-1}$. Values are means of duplicate assays.

\begin{tabular}{lccccc}
\hline \hline & \multicolumn{2}{c}{ PMN } & & \multicolumn{2}{c}{ Monocytes } \\
\cline { 2 - 3 } \cline { 5 - 6 } Treatment & Resting & $\begin{array}{c}\text { Phago- } \\
\text { cytosing }\end{array}$ & & Resting & $\begin{array}{c}\text { Phago- } \\
\text { cytosing }\end{array}$ \\
\hline None & 3 & 59 & & 7 & 44 \\
LPS $(20 \mu \mathrm{g})$ & 8 & 73 & & 26 & 57 \\
PG $(15 \mu \mathrm{g})$ & 36 & 83 & & 20 & 67 \\
\hline
\end{tabular}

al., 1988; De Souza \& Koury, 1991). In the present investigation, we describe some relevant biological effects exerted in vitro by PG extracted from a strain of Leptospira interrogans, which are typical of the biologically active peptidoglycans. We demonstrate here that leptospiral PG is capable of activating human complement at very low concentrations when compared to peptidoglycans of other micro-organisms; in fact only $8 \mu \mathrm{g} \mathrm{PG} \mathrm{ml}^{-1}$ were sufficent to reduce by $50 \%$ the haemolytic activity of complement, whereas $200 \mu \mathrm{g} T$. pallidum PG (Umemoto et al., 1981) and about $0.5 \mathrm{mg}$ of Staph. aureus PG (Peterson et al., 1978) were required to produce a comparable effect. Such a finding indentifies PG as a cellular fraction responsible for the activation of complement, which was known already to be triggered by whole leptospires (Cinco \& Banfi, 1983). Since leptospires are known to be complement resistant (Cinco \& Banfi, 1983), we may postulate that complement activation by $P G$ acts as an indirect factor in the stimulation of phagocytic cells.

A direct stimulation of PMN and monocytes was also observed using similar amounts of PG, since $15 \mu \mathrm{g}$ PG increased the release of oxidative products from the cells during both phagocytosis and the resting state, and at a rate which is slightly higher than that obtained with $E$. coli LPS. Moreover PG enhanced PMN uptake of Staph. aureus. Taken together, these direct and indirect stimulatory effects on the leucocytes may result in an amplification of the phagocytic process. Unlike peptidoglycans of other spirochaetes (Umemoto et al., 1981; Beck et al., 1990) but in agreement with results obtained for the majority of peptidoglycan preparations (StewartTull, 1980), leptospiral PG was mitogenic for peripheral mononuclear cells. This in vitro observation, previously reported also for leptospiral LPS (Shimizu et al., 1987), suggests an in vivo role for leptospiral PG in activation of the immunocompetent system.

In summary, the biological activities of leptospiral PG reported in this study suggest that it plays a role in modulating the function of immunocompetent cells. We recently demonstrated that PG, but not LPS, both extracted from Leptospira strain Teramo, was able to trigger a proadhesive effect on endothelial cells towards PMN (A. Dobrina and others, unpublished data): this could suggest that PG is involved also in generalized vasculitis and the late symptoms which can develop in leptospirosis. These biological characteristics of PG and the few documented effects induced by leptospiral LPS may account for the multi-systemic course of leptospirosis. That both agents activate complement and stimulate leucocytes could contribute to the amplification of the biological response induced by spirochaetes.

\section{References}

Alston, J. M. \& Broom, J. C. (1958). Leptospirosis in Man and Animals. Edinburgh and London: E. \& S. Livingstone.

Arean, V. M., Sarasin, G. \& Green, J. H. (1964). The pathogenesis of leptospirosis: toxin production by Leptospira icterohaemorrhagiae. American Journal of Veterinary Research 25, 836-843.

azuma, I., Taniyama, T., Yamamura, Y., Yanagihara, Y., Hattori, Y., DA Yasu, S. \& Mifuchi, I. (1975). Chemical studies on the cell walls of Leptospira biflexa, strain Urawa and Treponema pallidum strain Reiter. Japanese Journal of Microbiology 19, 45-51.

BECK, G., BeNACH, J. L. \& HABICHT, G. S. (1990). Isolation preliminary chemical characterization and biological activity of Borrelia burg. dorferi peptidoglycan. Biochemical and Biophysical Research Communications 167, 89-95.

Cinco, M. \& Banfi, E. (1983). Activation of complement by leptospires and its bactericidal activity. Zentralblatt für Bakteriologie und Hygiene (Abt. I, Orig. A) 254, 261-265.

Cinco, M., Banfi, E., Giani, M., Gundelach, M. L. \& Galanos, C. (1988). Chemical and biological properties of a phenol-water extract from Leptospira interrogans. Evidence for the absence of lipopolysaccharide. Infection 16, 238-241.

De Souza, L. \& Koury, M. C. (1991). Isolation and biological activities of endotoxin from Leptospira interrogans. Canadian Journal of Microbiology 38, 284-289.

Gerdes, J., Lemke, H., Baisch, H., Wacker, H. H., Schwab, U. \& STEIN, H. (1984). Cell cycle analysis of a cell proliferation associated human nuclear antigen defined by the monoclonal antibody $\mathrm{Ki}-67$. Journal of Immunology 133, 1710-1715.

GINGER, C. D. (1963). Isolation and characterization of muramic acid from two spirochaetes: Borrelia duttoni and Leptospira biflexa. Nature, London 13, 159.

Johnson, R. C., Walby, J., Henry, R. A. \& Auran, N. E. (1973). Cultivation of parasitic leptospires: effect of pyruvate. Applied Microbiology 26, 118-119.

Joseph, R., Holt, S. C. \& Canale-Parola, E. (1973). Free-living anaerobic spirochetes. Journal of Bacteriology 115, 426-435.

Harrison, R. A. \& Lachmann, P. J. (1986). Complement technology. In Handbook of Experimental Immunology, pp. 39.20-39.25. Edited by D. L. Weir. Oxford: Blackwell Scientific Publications.

Kotani, S., Tsujmoto, M., Koga, T., Nagao, S., Tanaka, A. \& Kawata, S. (1986). Chemical structure and biological activity relationship of bacterial cell walls and muramyl peptides. Federation Proceedings 45, 2534-2540.

MacKenzie, S. L. \& Tenashuk, D. (1979). Quantitative formation of $\mathrm{N}(\mathrm{OS})$ heptafluorobutyryl isobutyl aminoacids for gas chromatographic analysis. Journal of Chromatography 173, 53-63.

MClean, I. W. \& NAKANE, P. K. (1974). Periodate-lysineparaformaldehyde fixative: a new fixative for immunoelectron microscopy. Journal of Histochemistry and Cytochemistry 22, 1077-1083.

Perticarari, S., Presani, G., Mangiarotti, M. A. \& Banfi, E. (1991). 
Simultaneous flow cytometric method to measure phagocytosis and oxidative products by neutrophils. Cytometry 12, 687-693.

Peterson, P. K., Wilkinson, W. J., Kim, Y., Schmeling, D., Douglas, S. D. \& QuIE, P. G. (1978). The key role of peptidoglycan on the opsonization of Staphylococcus aureus. Journal of Clinical Investigation 61, 597-609.

Pillot, J. \& RyteR, A. (1965). Structure des spirochetes. I. Etude des generes Treponema, Borrelia et Leptospira au microscopie electronique. Annales de l'Institut Pasteur 108, 791-804.

Schwarting, R., Gerdes, J., Niehus, J., Jaeschke, L. \& Stein, H. (1986). Determination of the growth fraction in cell suspension by flow cytometry using the monoclonal antibody Ki-67. Journal of Immunological Methods 90, 65-70.

SCHLEIFER, K. \& KandLER, O. (1972). Peptidoglycan types of bacterial cell walls and their taxonomic implications. Bacteriological Reviews 36, 407-477.

SCHLEIMER, R. P. \& Rutledge, B. K. (1986). Cultures of human vascular endothelial cells acquire adhesiveness for neutrophils after stimulation with interleukin-1, endotoxin and tumor-promoting phorbol diesters. Journal of Immunology 136, 649-657.
Shimizu, T., Matsusaka, E., Takayanagi, K., Tagakura, N., Masuzawa, T., IWamoto, Y., MORITA, T., MifuchI, I. \& YANAGIHARA, Y. (1987). Biological activities of lipopolysaccharide like substance (LLS) extracted from Leptospira interrogans serovar canicola strain Moulton. Microbiology and Immunology 31, 727-735.

STEWART-TULL, D. E. S. (1980). The immunological activities of bacterial peptidoglycans. Annual Review of Microbiology 34, 311-340.

TSaI, C. M. \& Frasch, C. E. (1982). A sensitive silver stain for detecting lipopolysaccharides in polyacrylamide gels. Analytical Biochemistry 119, 115-119.

Umemoto, T., Ota, T., Sagawa, H., Kato, K., Takada, H., Tsujimoto, M., Kawasaki, A., Ogawa, T., Harada, K. \& Kotani, S. (1981). Chemical and biological properties of a peptidoglycan isolated from Treponema pallidum kazan. Infection and Immunity 31 , $767-774$.

Yanagihara, Y., Kamisango, K., Yasuda, S., Kobayashi, S., Mifuchi, I., Azuma, I., Yamamura, Y. \& Johnson, R. C. (1984). Chemical composition of cell walls and polysaccharide fractions of Spirochete. Microbiology and Immunology 28, 535-544. 